\title{
DVQ: A DTD-DRIVEN VISUAL QUERY INTERFACE FOR XML DATABASE SYSTEMS
}

\author{
Long Zhang, Shihui Zheng, Aoying Zhou \\ Computer Science \& Engineering Department \\ Fudan University, Shanghai, China \\ \{lzhang0, shzheng0, ayzhou\}@fudan.edu.cn
}

\author{
Hongjun Lu \\ Computer Science Department \\ Hong Kong University of Science \& Technology, Hong Kong, China \\ luhj@cs.ust.hk
}

\begin{abstract}
XML has been recognized as a promising language for data exchange over the Internet. A number of query languages have been proposed for querying XML data. Most of those languages are path-expression based. One difficulty in forming path-expression based queries is that users have to know the structure of XML data against which the queries are issued. In this paper, we describe a DTD-driven visual query interface for XML database systems. With such interface, a user can easily form path-expression based queries by clicking elements in the DTD tree displayed on the screen and supplying conditions if necessary. The interface and the query generation process are described in detail.
\end{abstract}

Keywords: XML, DTD, visual query, path expressions

\section{INTRODUCTION}

The Extensible Markup Language (XML) is an emerging standard for data representation and exchange on the Internet. There is a consensus among the researchers that XML is as an easy-to-write, easy-to-parse language to exchange data in a variety of applications on the Internet. Currently, most business data is stored in relational or object-relational systems. It will continue to be so since the matured relational technology provides excellent queriability, scalability and availability. It becomes a natural choice to store XML documents in relational systems to make use of such advantages and hopefully 
ease the difficulties in integrating XML documents into other applications. Large amount of research work has been conducted to study issues related to storing XML documents using relational database management systems ( Shanmugasundaram et al., 1999; Deutsch et al., 1999; Shanmugasundaram et al., 2000; Manolescu et al., 2001). Storing an XML document into a relational database system requires to map the document into relational tables. In other words, a relational schema must be defined. Yoshikawa and Amagasa categorize such XML-Relation mapping into two categories: structuremapping-based approach and model-mapping-based approach (Yoshikawa and Amagasa, 2001). The structure-mapping-based approach generates a relational schema from an XML document based on its logical structure described by its DTD (Document Type Descriptor). The model-mapping-based approach generates a relational schema for an XML document based on its representation model (e.g. trees) without understanding its logical structure.

To query XML data, several XML query languages were proposed including LOREL (Abiteboul et al., 1997), XML-QL (Deutsch et al., 1998), XPath ( Clark and DeRose, 1999) and XQuery (Chamberlin et al., 2001). Although those query languages differ from each other in ways of expressing queries, one of the common features of XML queries is that they are mainly path expression based, which is rather different from set-oriented relational query languages, such as SQL.

Compared to querying relational database using SQL, querying XML data is more difficult from the users' viewpoint since users need to know the structure of the data. When XML data is stored in relational systems, this becomes much more difficult, especially when structure-mapping based approach is used for schema mapping as the original structure may not be well reflected by the relational schema. While storage of XML and related query processing techniques received well attention, the efforts in providing users with facilities to ease the difficulty of writing path-expression based XML query are very limited. The DataGuide (Goldman and Widom, 1997; Goldman and Widom, 1998) interface in Lore system can generate simple LOREL (Abiteboul et al., 1997) queries by exploring the DataGuide of semi-structured data. But it can only specify simple path expressions, and set conjunctive conditions on only a unique path. Both the BBQ (Munro et al., 2000) visual interface of MIX and EquiX (Cohen et al., 1999) interface display the DTD structure of XML data to facilitate user to browse and formulate query. But they cannot construct arbitrary regular path expressions and complex constraint conditions too.

In this paper we present DVQ, a DTD-driven visual query interface for an XML-Relational database system VXMLR (Zhou et al., 2001). VXMLR adopts a structure-mapping based approach to map XML data into relational tables managed by a commercial RDBMS. Its query interface, DVQ, displays the structure of the stored XML data on the screen. By simple clicking the 
nodes in the DTD structure and entering related conditions, a user can easily form path expressions. DVQ then generates the path expression based queries automatically. The query results are represented by XSLT and delivered to users through DVQ. With DVQ, VXMLR users navigate the nested structure of the stored XML data, form query and browse the result documents through DVQ seamlessly. The unique features of DVQ include the following.

- It provides users with a graphical interface so that complex queries can be formed by users' simple GUI actions. Not only XML experts, but also ordinary users can specify query without the knowledge of the XML query languages.

- It is powerful enough to specify regular path expressions with wildcards and any complicate conditions with conjunction, disjunction and negation. At the same time, the WYSWYG (What You See is What You Get) feature of DVQ makes query formulation rather straightforward.

- In addition to enter queries, DVQ also provides facilities for users to navigate through the XML structure and to view the intermediate results of the major steps in query processing.

- It is a separate module running at the client side and driven by DTD of original XML data. That is, it is independent on the underlying XMLrelational mapping schema. So, it is a portable module that can be used in any XML database system.

The remainder of this paper is organized as follows. In Section 2, we introduce some background information. In Section 3, we describe the architecture of DVQ. Section 4 describes how DVQ forms path expressions with set constraint conditions using examples. In Section 5, we present DVQ's function of monitoring XML query execution and displaying the query results. Finally, conclusions are presented in Section 6.

\section{BACKGROUND INFORMATION}

In this section, we introduce some background information about XML data and XML queries.

\subsection{A DTD Sample}

Unlike HTML documents where their structures are usually unknown, the data type definition (DTD) of an XML document describes the nested relationships between data elements in the document. A DTD example is shown in Figure 1, and we will use it as running example in the following discussion.

The DTD in Figure 1 describes XML documents about the information of laboratories. There are two basic constructs in XML documents, element and 
attributes, indicated by !ELEMENT and ! ATTLIST, respectively. Elements in XML documents can be nested. Each document has a unique root element, which is labinformation in our example. It contains three sub-elements, labname, project, and member. Character * after project indicates that a laboratory element instance can have zero or more projects. + indicates that a laboratory element instance may have one or more members. Each member element has a unique ID attribute, and sub-elements name, email, URL, publication and project. The sign ? after URL indicates that this element is optional, that is, a member instance may not have a URL. The contents of an element is denoted by \#PCDATA. The other portion of the DTD can be interpreted in a similar way. Note that member and project are defined recursively. That is, a member element can have project as sub-element, and vice versa.

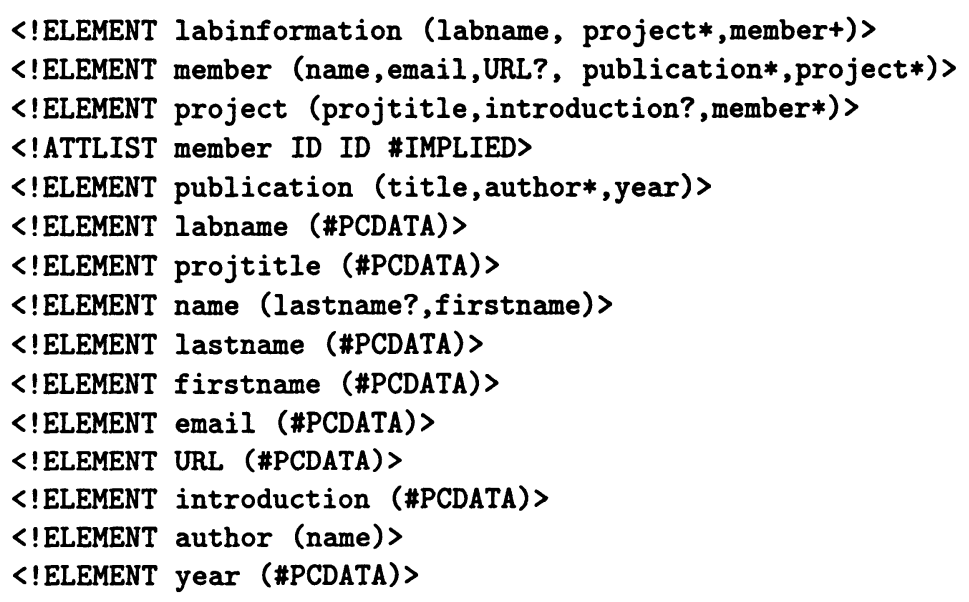

Figure 1. A Sample DTD.

\subsection{Path Expressions}

We can see from the DTD example that data elements in an XML document are nested to form a tree structure (a graph with IDREF). Figure 2 depicts an example document and its graphical representation, data graph. With such a structure, we can represent an element by the path from the root of the tree to the element. If we adopt the dot notation to denote the parent-child relationship, the path for a members name can be expressed as project .member .name, for example. In general, the path expressions can be more complex. If $r, r_{1}$, and $r_{2}$ are elements or attributes, a path expression has the form the root of $r=(r) *|(r)+|(r) ?\left|r_{1} . r_{2}\right|\left(r_{1} \mid r_{2}\right)|\#|$ name

Where $*,+, ?$ mean 0 or more, 1 or more, and 0 or 1 , occurrences, respectively. Concatenation $r_{1} . r_{2}$ is used to form a path from $r_{1}$ to $r_{2}$. 
Alternation “| " stands for disjunction. Wildcard “\#” denotes arbitrary occurrences of any regular expressions. We distinguish two types of path expressions: simple path expression (SPE) and regular path expression (RPE). Simple path expressions are path expressions that consist of only element or attribute names such as labinformation.project.member.name. Regular path expressions are path expressions that contain regular operators. For example, \#. (project.member)*. name is a RPE.
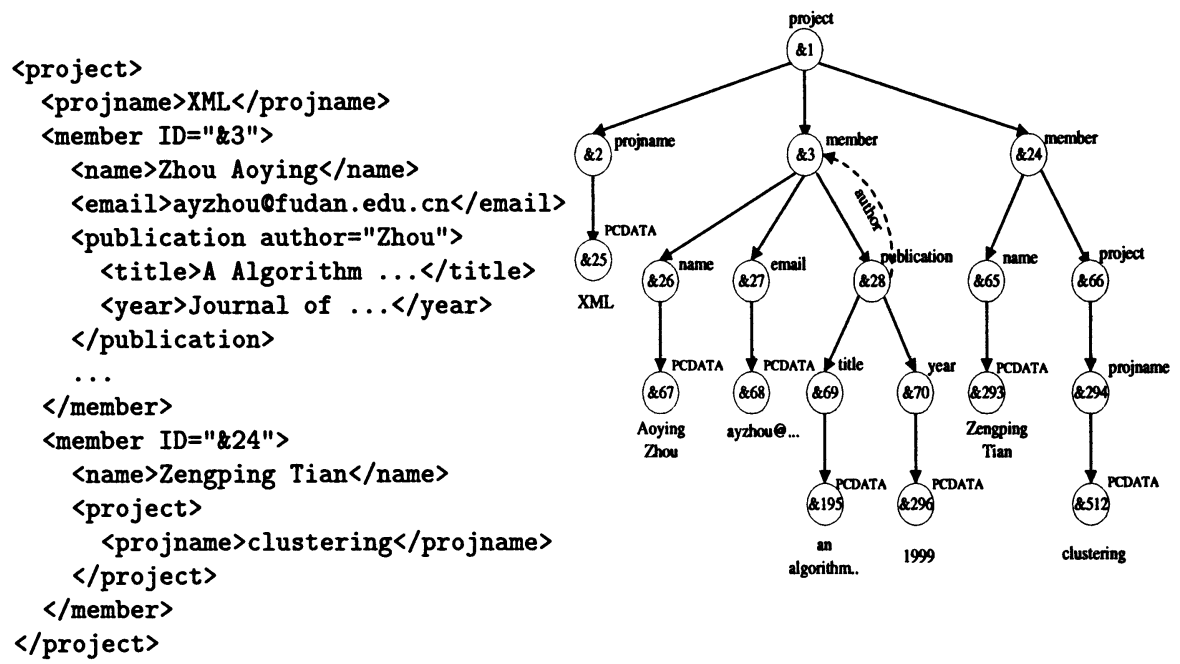

Figure 2. An XML document and its data graph.

\subsection{VXMLR: A Visual XML-Relational Database System}

The detailed description of VXMLR is out of the scope of this paper. To illustrate the context where DVQ works, we depict the architecture of VXMLR as shown in Figure 3. In VXMLR, XML documents are stored in a relational database system, managed by a relational DBMS. An input XML document is first parsed into a DOM tree. At the same time, the DTD for the document is extracted. The document tree is then mapped to relational tables and stored in the database. User issue queries through DVQ, the visual interface. Those queries are then transformed into SQL statements ready to submit to the underlying relational DBMS. To generate efficient SQL statements from path expression based queries, VXMLR maintains some statistics of data and a path directory. Both the statistics and the path directory are used in the query rewriting process to reduce the number of SQL statements and simplify join conditions. The query results returned from DBMS are constructed and expressed using XSL, which are then delivered to the user through DVQ. 


\section{DVQ: THE VISUAL QUERY INTERFACE}

VXMLR system was implemented on the top of Microsoft SQL Server. The server side program is implemented in $\mathrm{C}++$. The client side program, i.e., the visual query interface, which runs on a browser, is written in Java. Figure 4 shows the reference architecture of DVQ.

The portion upper the dashed line is the client side of VXMLR, while the below portion is the

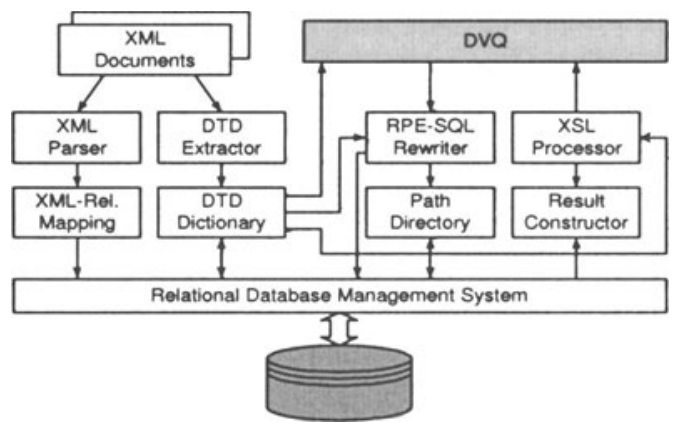
server side. The system works as following: at first, once a DTD descriptor is selected by user, the DTD Sender transfers the encoded DTD information to DVQ by way of CGI. Next, the DTD Receiver at the client side receives the decoded DTD and the DTD Tree Generator parses it into hierarchical structure, which then is displayed in Query Interface. Once user has constructed the query and clicked the Submit button, The Query Generator module generates path expression query automatically. The Query Sender module then sends the generated query to server. At the server side, the Query Receiver decodes the query and submits it to the Query Engine of VXMLR. The Query Engine translates the query into efficient SQL statements, which is then executed by the underlying RDBMS. VXMLR Query Engine constructs the result document using the result relational tables returned by RDBMS. Finally, the result document is represented by the XSL Processor and transferred to the browser for displaying.

Figure 5 is the screen dump (main window) of DVQ interface. It consists of two panels and some buttons. The left panel displays the DTD structure of XML data to be queried. What shown is the sample DTD in Figure 1 as a hierarchical directory, where elements and attributes are represented by their names, preceded with a diamond or a dot, respectively. Sub-elements or attributes are

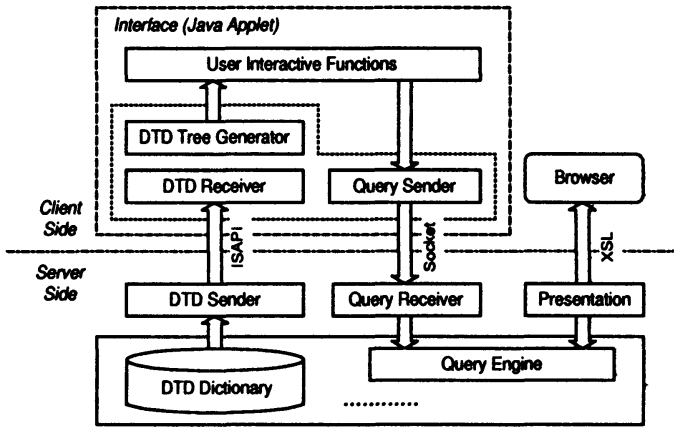

Figure 4. The reference architecture of DVQ. 
expressed as the children of their parent node. User can click the icon before an element to explore or collapse its subtree. Recall that project and member are defined recursively, which is represented by "project-member" and "member-project" in the DTD panel. The DTD panel provides an easy way for user to navigate the nested structure of the stored XML data conforming to the DTD. To the right of the DTD panel is two tabbed panels, Selection

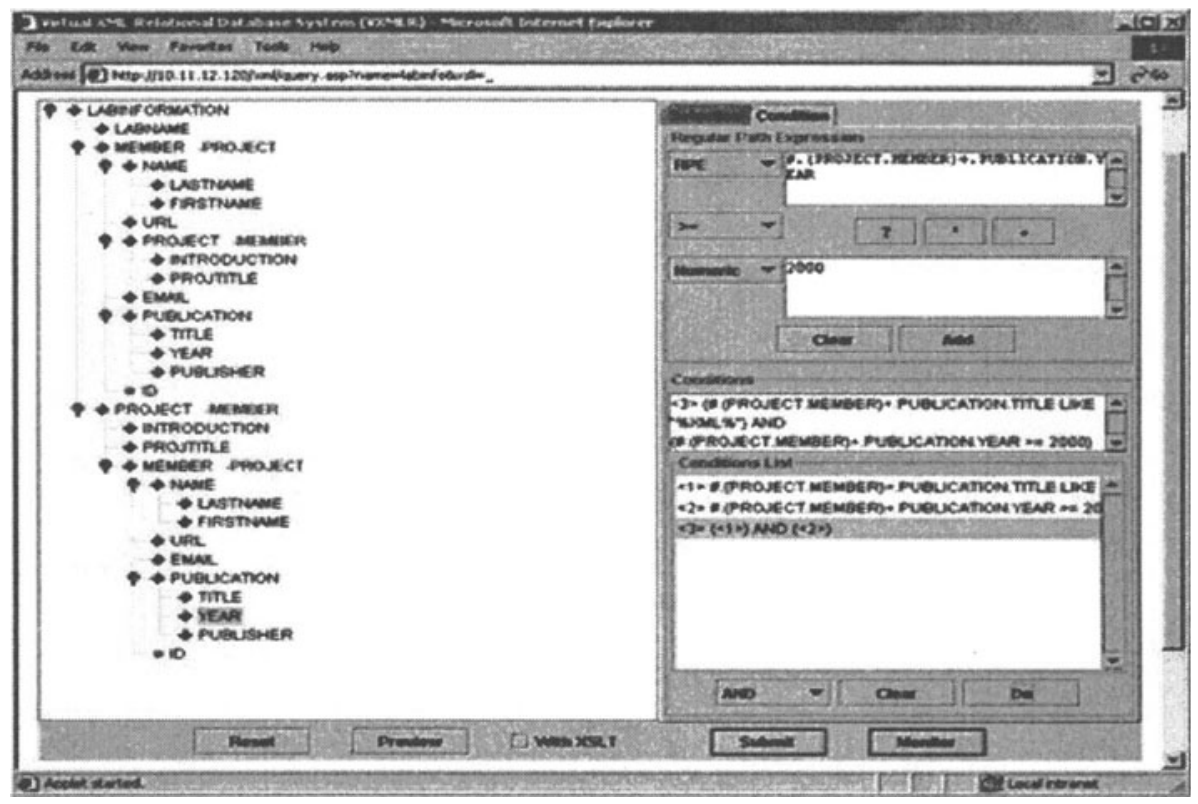

Figure 5. The DVQ interface.

and Condition. The Selection panel is used to form the target items (i.e. a list of path expression) to be retrieved. The Condition panel is for user entering the conditions that the retrieved items must satisfy. The basic components of both panels are path expression.

At the bottom of the main window are four buttons and a check box. The leftmost is the Reset button, which lets user to clear up the constructed items in the Selection and Condition panels. The Preview button brings up a dialog that displays the generated query for user to preview. To the right of the Preview button is a XSLT check box, which indicates the query result will be returned as plain XML file or XML page expressed by XSL. To the right of the XSLT check box is the Submit button, which triggers the query to be submitted to the VXMLR server for executing. When the right most Monitor button is pushed, an XML page will displayed that shows the elapsed time and query statements in each step of query processing. 


\section{VISUAL QUERY FORMATION}

A user query session in VXMLR consists of four steps: First, browses the DTD structure and forms the output items by clicking nodes in the DTD structure and set constraints through the Condition panel. Second, after a user completes the specifications of the output items and constraints, a query in the form of path expressions is generated. Third, the generated query is submitted to the VXMLR server for executing. Finally, the result is returned from VXMLR server to the interface for user to browse.

\subsection{Form Path Expressions}

We first introduce the Selection panel and then show how to formulate path expressions in the select part of a query. Figure 6 shows the DTD panel and

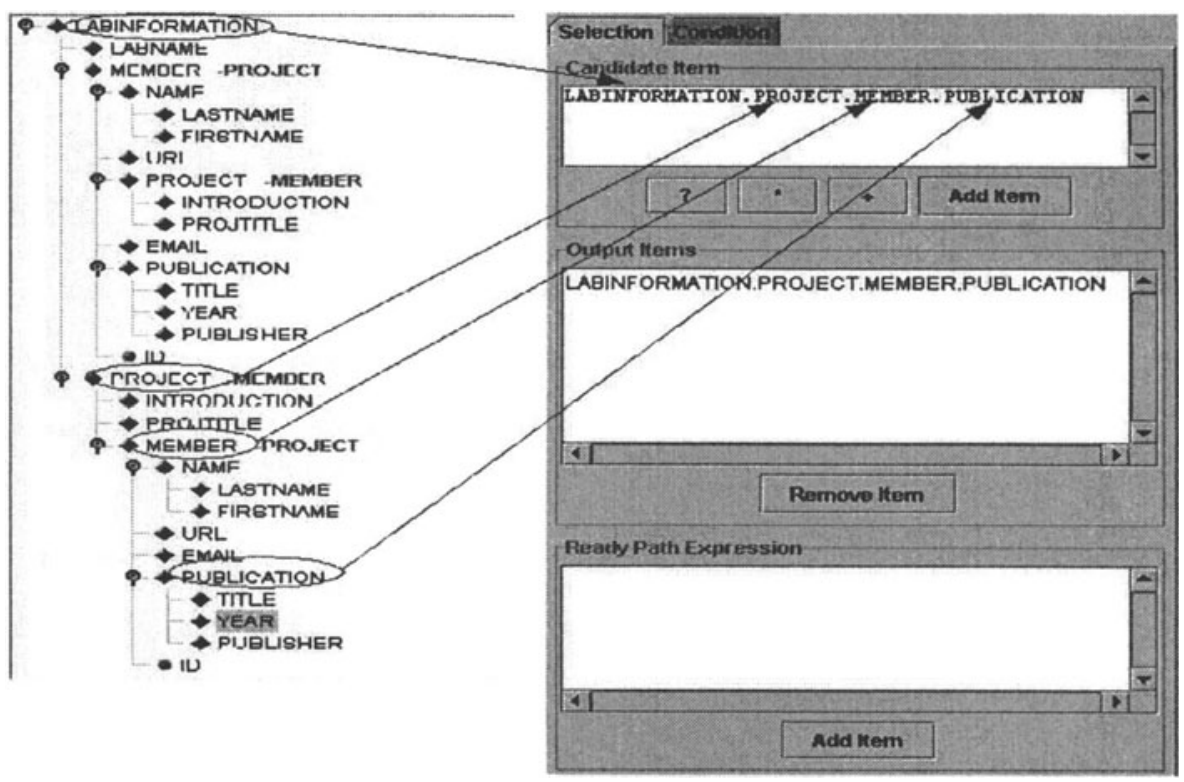

Figure 6. Specifying simple path expression by SELECT panel.

Selection panel. The Selection panel consists of three text boxes, Candidate Item, Output Items, and Ready Path Expression, and some buttons. The first text box on the top of Selection panel, Candidate Item, is used for showing the path expression being constructed. There are four buttons under the Candidate Item text box. The first three buttons are regular operator buttons labelled with "?", “*”, and "+" respectively, which trigger those regular operators been added to the current path expression shown in the select text box. To the right of the regular operator buttons is the Add Item button, which brings up the path 
expression in the Candidate Item text box added into the Output Items text box under those buttons.

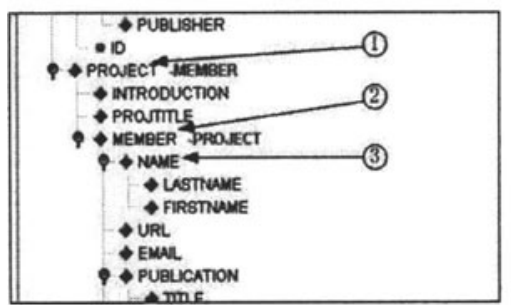

(1)

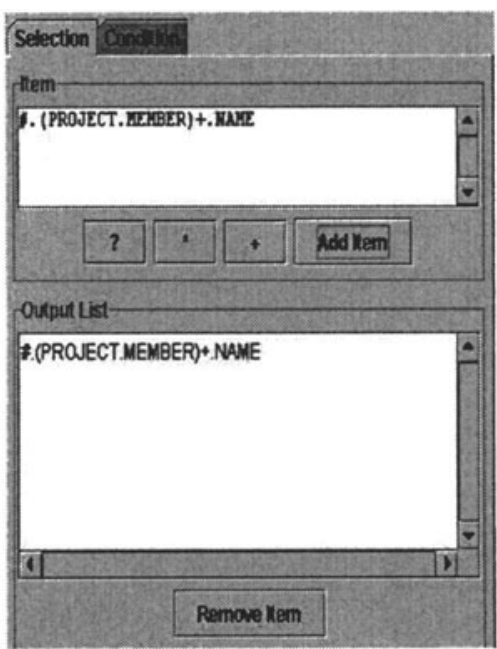

(2)

Figure 7. Example for specifying regular path expressions by DTD panel (1) and SELECTION panel (2).

Now, we show by example how to form simple path expressions. A simple path expression is constructed in a natural way by clicking the nodes in the DTD panel following top-down links. Clicking a node and its children starting from the root triggers the name of the clicked nodes appended into the Candidate Item text box consecutively. Figure 6 shows that user clicks the nodes labinformation,project, member and publication consecutively. The generated path expression is SPE,

labinformation.project.member.publication

which is displayed in the Condition Item text box.

If the first clicked element is not the root element, or not the child of the element in the tail of the current path expression, then there is at least one element between the tail of the current path and the clicked element. A "\#" operator is inserted before it automatically. For example, as shown in Figure 7(1) step one, user clicks the project element first followed by element member and name element. The first clicked element, project, is not the root. Those actions form a regular path expression \#. project .member. name.

User can also insert the regular operators manually by first highlighting a segment in the path expression followed by clicking the corresponding operator button. The operator is then added on the path segment automatically. In Figure 7(2), highlighting path segment project.member followed by clicking "+" button forms a regular path expression \#. (project.member)+.name. 
Now user clicks the Add Item button. It triggers the current path expression in the Candidate Item text box appended to the Output text box. User can specifies target items and add them into the output items continuously.

Alternatively, user can also use the Remove Item button under the Output text box to remove one or more path expressions from the Output Item text box. At last, the path expressions in the Output Item text box will be used to construct the select part of the query.

\subsection{Set Constraints on Output Items}

Constraints can be placed on the paths in output items by Condition panel. Figure 8 shows the Condition panel, it consists of three text boxes, three pop-menus and several buttons. Same as the Selection panel, the Path Expression text box on the top of the Condition panel and the three regular operator buttons under it are for user to form path expressions, on which constraints are then replaced. The below part of the Condition panel is two text boxes labelled with "Conditions" and "Condition List", which let the user to combine multiple constraints to form complex conditions.

To the left of the "?" operator button is the comparator popup menu, which consists of a set of basic comparators ("=", " $>$ ", ">=", "<", "<=", "LIKE", “IN"). Below the comparator popup menu is the Type popup menu, which indicates the type of the expression. The type of an expression can be a numeric, a string, a Boolean, or a path expression. In VXMLR, each ele-

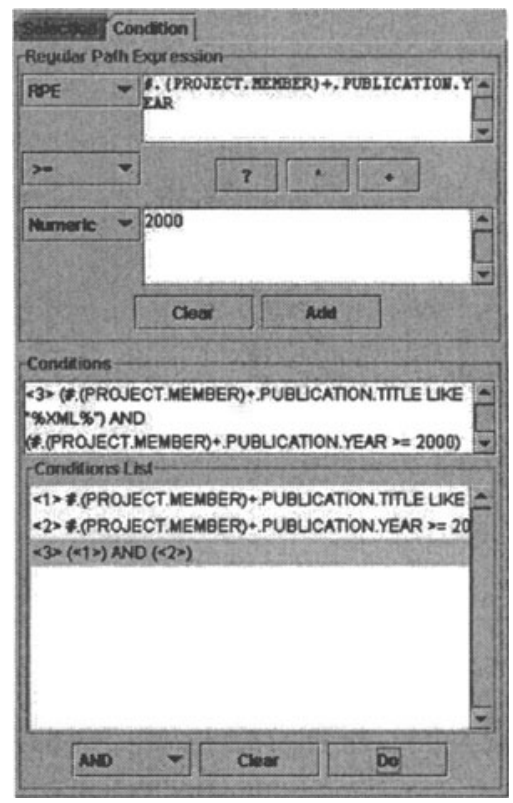

Figure 8. Forming constraints with CONDITION panel. ment is stored as an attribute with string type in relational database. The comparator and type popup menus enable user to flexibly treat an element as other types. For example, user can specify a comparison between target element "year" with an integer "2000". In VXMLR, the comparison is implemented by casting the attribute in the relation as a numeric type. To the right of the Type popup menu is the Expression text box. User can use it to input an expression, which forms a condition comparing the path expression in the Path Expression text box with an expression, or input another path expression by clicking the nodes in the DTD panel, which form a condition that joins two paths. In Figure 8 , user first forms this path expression "\#. (project.member)+.publication.year" in the Path Expression text box by clicking the corresponding elements and " + " button. Next, user selects 
the comparator " $>=$ " and "NUMERIC" type, and inputs an integer values "2000" in the Expression text box. Finally, the following constraint condition

\#. (project .member) +. publication . year $>=2000$

is formed. At this point, user can click the "ADD" button under the Expression text box to add the above condition into the Condition List text box or withdraw the formed condition by clicking the Clear button under the expression text box. In the Condition List text box in Figure 8, the condition has been added into the Condition text box.

DVQ is powerful enough to construct arbitrary complex conditions with "AND", "OR" and "NOT" predicates. Figure 8 also shows how to form complex conditions using the Predicate popup menu at the bottom of the Condition panel. User first highlights the two conditions have been added to the Condition text box, then selects the "AND" predicate from the Predicate popup menu. Those actions bring up the two conditions in the Condition text box are connected by "AND" predicate and the following conjunctive condition,

\#. (project.member)+.publication.title LIKE '\%XML\%'

AND \#. (project.member)+.publication. year $>=2000$

is formed. Continuing to add expressions and predicates, arbitrary nested complex condition composed of conjunction, disjunction and negation can be composed also. The generated conditions are simultaneously displayed in the Conditions text box above the Condition List text box. To the right of the predicate popup menu of the two buttons are labelled with "Clear" and "Add", which bring up the conditions in the Condition text box are cleared or added into the where part of the formed query.

\subsection{Executing Query and Browsing Result}

After a user specifies the path expression and the related conditions as shown in Figure 7 (2) and Figure 8, DVQ will form the query automatically. User can preview the generated query by clicking the Preview button in the right part of the main window.

Figure 9 shows the dialog triggered by the click action, which displays the generated query. Note that variables are added into the queries. DVQ converts the query into the

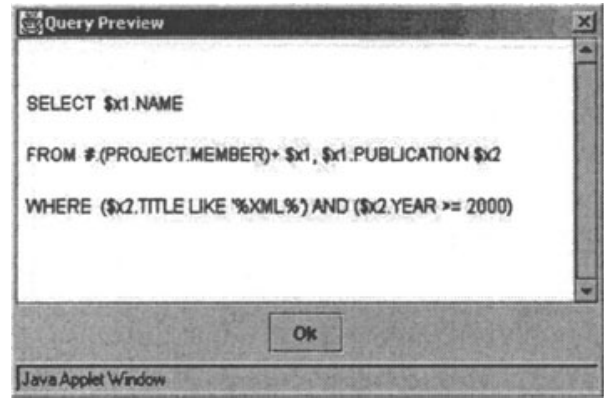

Figure 9. The preview dialog box displaying the generated query. forms of Figure 9 automatically. We just sketch out the main idea for conversion and do not go into the details for query conversion here: the query generator first finds all of the path prefixes 
of those path expression appearing in the output items and constraint conditions, next, assigns each common path prefix a variable and place those common prefixes in the FROM clause. Then replaces all common prefixes with variable in all of those path expressions. Finally, states the constraints in the WHERE clause and puts the retrieved items in the SELECT clause. For our example, \# . (project .member) + and publication are two common prefixes, which are assigned variables $\$ \mathrm{x} 1$ and $\$ \mathrm{x} 2$ respectively. The SELECT clause consists of the target item \$x1.member.name and WHERE clause consists of “\$x2.title LIKE' \%XML\%' and $\$ \times 2$. year $>=2000$ ".

The generated query can be submitted to the VXMLR sever by click the Submit button. Then the query session enters into the third step: VXMLR server translates the query into SQL statements and submits it to RDBMS, then transforms the results into XML documents. If the XSLT check box is selected, then the XSLT sheet of the document is also returned. Finally, the result document is displayed in the browser and delivered to user. For example, the following query,

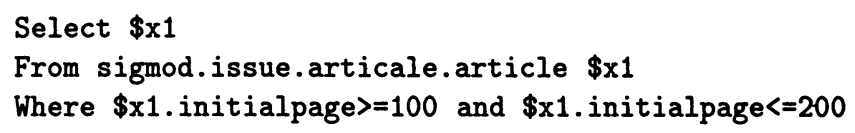

retrieves the articles with initial page above 100 in SIGMOD RECORD, and the result document represented by XSLT is shown in Figure 10.

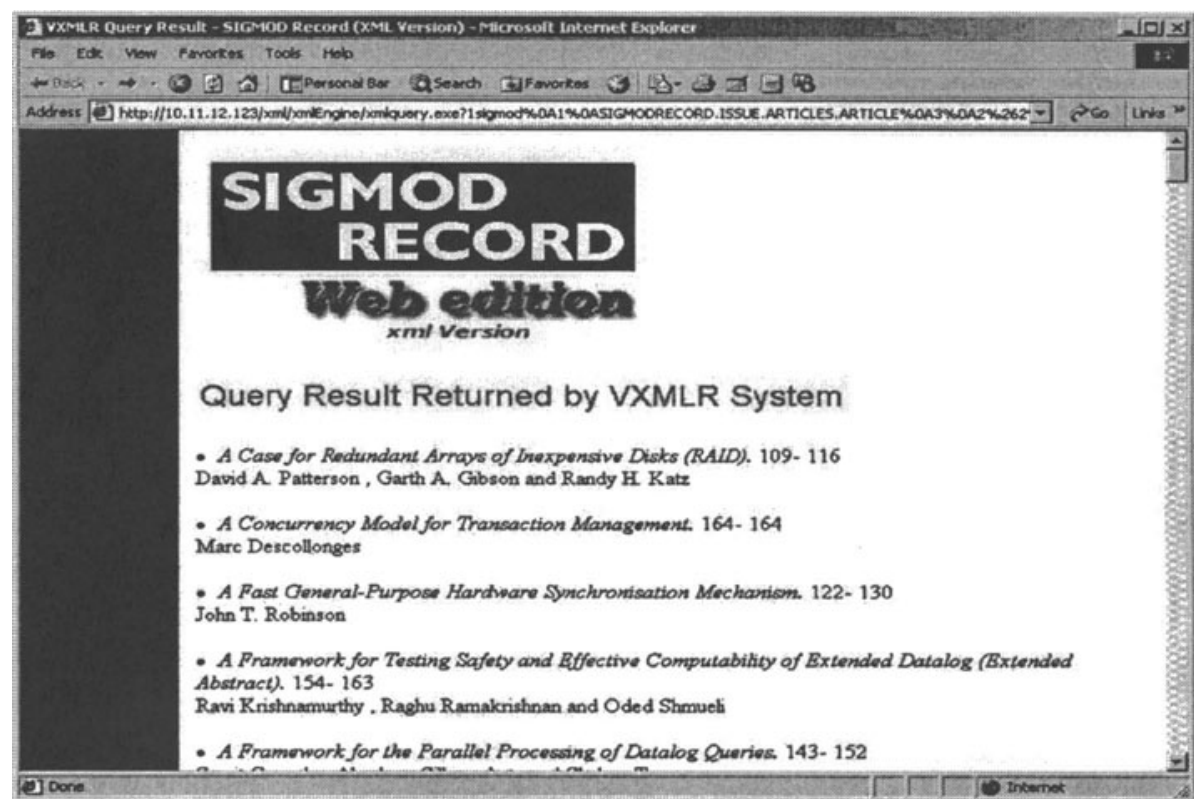

Figure 10. The result document represented by XSL. 


\section{MONITORING FACILITY}

Querying XML data stored in relational systems is a relatively complex process. The user formed path expression queries have to be translated into SQL statements first. When the user query is a regular expression query, it needs to be transformed to simple path expression queries first. With the monitoring facility of DVQ, user can view the results of those intermediate results, in addition to time used for each step.

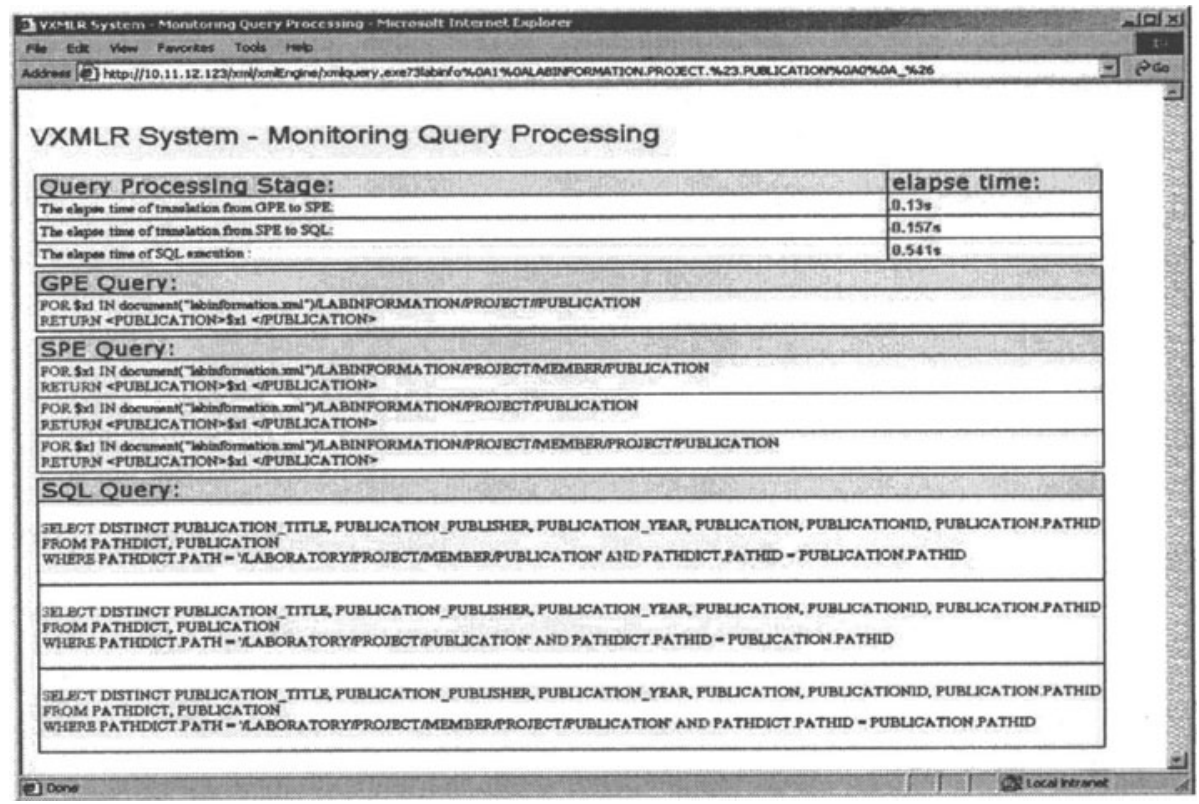

Figure 11. Browing query translation

Figure 11 shows a screen dump of execution of a query that selects all of the publications reachable from the project node via zero or more edges. That is, select labinformation.project.\#.publication

It can be seen that DVQ displays the following information: the elapsed time of each main query processing stage and their results, the original regular path expression query, the corresponding simple path expression queries, and the SQL statements executed by the underlying DBMS.

For the example shown in Figure 11, the original RPE query is expanded to three simple path expressions.

select labinformation.project.member.publication
union select labinformation.project.publication
union select labinformation.project.member.project.publication.

A simple path expression can be straightforwardly translated into joins between parent element and child element in SQL statement. With the path directory that materializes the paths from the root to elements existing in the data, 
a simple path expression can be translated into a rather simple SQL query. For example, SPE query

select labinformation.project.member.publication

is translated into the following SQL statement.

select labinformation.project.member.publication

from PathDirectory, publication

where PathDirectory.path="abinformation.project.member.publication" and PathDirectory pathid=publication . pathid

\section{CONCLUSIONS}

In this paper, we presented DVQ, a DTD-driven visual query interface of a prototype XML databases system, VXMLR. DVQ provides users a graphical interface for navigating XML structure, forming complex path expression queries, submitting queries for execution, browsing query results and monitoring the main steps of a query session.

The current version of DVQ only displays one DTD, and user can only query single document. We are extending this version so that users can query across related documents with different DTDs.

\section{REFERENCES}

S. Abiteboul, D. Quass, J. Mchugh, and et al. The Lore Query Language for Semistructured Data, International Journal on Digital Libraries, 1(1): 68-88, April 1997.

J. Clark and S. DeRose. XML path language (XPath), W3C Recommendation 16 Nov. 1999, http://www.w3.org/TR/xpath, 1999.

D. Chamberlin, D. Florescu, J. Robie, J. Simeon, and et. al. XQuery: A query Language for XML, Technical report, World Wide Web Consortium, Feb. 2001.

Available from http://www.w3.org/TR/xquery.

Michael J. Carey, Laura M. Haas, Vivekananda Maganty, John H. Williams: PESTO : An Integrated Query/Browser for Object Databases, Proceedings of the Twenty-Second International Conference on Very Large Data Bases, 1996, 203-214.

S. Cohen, Y. Kanza, Y. Kogan, W. Nutt, Y. Sagiv, A. Serebrenik. EquiX Easy Querying in XML Databases, WebDB 1999: 43-48.

A. Deutsch, M. Fernandez, D. Florescu, and et al. XML-QL: A Query Language for XML, W3C Note, 1998. Available: http://www.w3.org/TR/1998/NOTE-xml-ql-19980819/

A. Deutsch, M. Fernandez, D. Suciu. Storing Semistructured Data with STORED, Proceedings of the 28th SIGMOD International Conference on Management of Data, May 1999.

Mary F. Fernandez, A. Morishima, D. Suciu. Efficient Evaluation of XML Middle-ware Queries, Proceedings of the International Conference on Management of Data, Santa Barbara, California. Jun. 2001.

R. Goldman and J. Widom. DataGuides: Enabling Query Formulation and Optimization in Semistructured Databases, Proceedings of the 23rd International Conference on Very Large Data Bases, Athens, Greece, August 1997, 436-445.

R. Goldman and J. Widom. Interactive Query and Search in Semistructured Databases, International Workshop on the Web and Databases, Valencia, Spain, Mar. 1998. 
I. Manolescu, D. Florescu, D. Kossmann. Answering XML queries on heterogeneous data sources, Proceedings of the International Conference on Very Large Data Bases, Roma, Italy, Sept. 2001.

A. Marian, S. Abiteboul, G. Cobena, L. Mignet. Change-Centric Management of Versions in an XML Warehouse, Proceedings of the 23rd International Conference on Very Large Data Bases, Roma, Italy, Sept. 2001.

J. McHugh, S. Abiteboul, R. Goldman, D. Quass, and J. Widom. Lore: A Database Management System for Semistructured Data, SIGMOD Record, 26(3): 54-66, Sept. 1997.

A. Mendelzon, G. Mihaila, T. Milo. Querying the World Wide Web, Int. J. on Digital Libraries, 1(1): 54-67. 1997.

K. Munroe, Y. Papakonstantinou. BBQ: A Visual Interface for Browsing and Querying XML, Proceedings of the Fifth IFIP Working Conference on Visual Database Systems, 2000.

B. Nguyen, S. Abiteboul, G. Cobena, M. Preda. Monitoring XML Data on the Web, Proceedings of the International Conference on Management of Data, Santa Barbara, California. June 2001.

J. Robie, J. Lapp, and D. Schach. XML Query Language (XQL), Available from http://www.w3.org/TandS/QL/QL98/pp/xql.html, Dec. 1998.

J. Shanmugasundaram, J. Kiernan, E. J. Shekita, and et.al. Querying XML views of relational data, Proceedings of the International Conference on Very Large Data Bases, Roma, Italy, Sept. 2001.

J. Shanmugasundaram, E. J. Shekita, R. Barr, and et.al. Efficiently Publishing Relational Data as XML Documents, Proceedings of the International Conference on Very Large Data Bases, Cairo, Egypt, September 2000, 65-76.

J. Shanmugasundaram, K. Tufte, C. Zhang, and et. al. Relational Databases for Querying XML Documents: Limitations and Opportunities, Proceedings of the International Conference on Very Large Data Bases Edinburgh, Scotland, 1999.

M. YoshiKawa and T. Amagasa. XRel: A path-based approach to storage and retrieval of XML documents using relational databases, ACM Transactions on Internet Technology, Volume 1, Number 1, 2001.

P. Veltri, S. Cluet, D. Vodislav. Views in a large scale XML repository, Proceedings of the International Conference on Very Large Data Bases, Roma, Italy, Sept. 2001.

A. Zhou, H. Lu, S. Zheng. and et. al. VXMLR: A Visual XML-Relational Database System, Proceedings of the International Conference on Very Large Data Bases, (Demonstration), Roma, Italy, Sept. 2001. 